\title{
A MAPPING METHOD OF INTEGRATING MULTI-SCALE RIVER THEMATIC MAPS
}

\author{
Wen Zhang ${ }^{\mathrm{a}}$, Cuiying Yue ${ }^{\mathrm{a}}$, Changlu Cui ${ }^{\mathrm{a}}$, Lingkui Meng ${ }^{\mathrm{a} *}$ \\ ${ }^{\mathrm{a}}$ School of Remote Sensing and Information Engineering, Wuhan University, Wuhan, Hubei, P.R. China \\ lkmeng@whu.edu.cn
}

Commission IV, WG IV/3

KEY WORDS: Multi-scale maps, Updating and Integrating, Features Completeness, Memory Size

\begin{abstract}
:
Small-scale maps are generally used in spatial analysis for fast calculation, but part of important features are missing due to its generalization level, which makes the analysis results less accurate. Therefore, it is necessary to improve feature completeness of smallscale maps. The goal of this paper is to put forward a mapping method of integrating the existing multi-scale river thematic maps. In order to achieve this goal, this paper proposed an algorithm for multi-scale line features matching by calculating the distance from node to polyline and an integrating algorithm by simplifying, shortening and merging the features from the original multi-scale thematic maps. The experimental results proved that the new map produced by the method proposed in this paper keeps the same scale as the original small-scale map and it is consistent with the original large-scale map in terms of feature completeness. The strategy proposed in this paper can be used to produce a new river thematic map concluding all the features that users need; moreover, the new map not only expresses features completely but also takes up less storage.
\end{abstract}

\section{INTRODUCTION}

Currently, in the field of basic geo-spatial database updating, scholars from GIS and cartography fields put forward an important research direction, that is, to update the maps with small scales by using the updated maps with large scales, and it is meaningful to make full use of the existing thematic maps with different scales. It not only improves the reusing rate of existing data, but also reduces the cost of data acquisition. Meanwhile, given the features completeness of the updated small-scale thematic map, it is necessary to integrate the multi-scale thematic maps, which is also the main goal of this paper.

There are three steps in the process of integrating multi-scale thematic maps, the first step is unifying the data's coordinate system, the second is data matching, and the third is data updating and integrating. It is easy to unify the data's coordinate system. The technology for data matching has been researched all the time by scholars at home and abroad. About data updating and integrating, Fu Zhongliang(2007) proposed a multi-scale data updating scheme which can automatically identify the changed data and realize linkage updating of multi-scale spatial data based on linkage-relation which constructed by geographical object. Wang pengbo(2009) analyzed the conception and connotation of multi-scale road data linkage updating, and put forward the strategy and technical details of multi-scale road data linkage updating. These methods can only update the smaller scale map but can't ensure the features

\footnotetext{
* Corresponding author
} 
completeness of the new updated map. The goal of this paper is not only to realize the updating of small-scale map, but also to make the updated map more complete by adding some features that were ignored because of the impact of its map scale. The new map can be used in the field of data spatial analysis, which will acquire more precise results and improve the efficiency of data processing.

This paper focused on the method of using the existing river thematic maps with different scales to produce a new river map. It proposed an algorithm for multi-scale line features matching by calculating the distance from node to polyline and an integrating algorithm by simplifying, shortening and merging features from original multi-scale thematic maps. Using these algorithms, a new map would be produced, which would keep the same scale as the original small-scale map and keep the same completeness as the original large one.

\section{METHOD}

\subsection{The Framework of Multi-scale Thematic Maps Integration}

In general, the process of multi-scale thematic maps integration includes three steps: namely coordinate system unifying, data matching, data updating and integrating. The purpose of unifying the data's coordinate system is to eliminate the differences between the multi-scale spatial data because of different sources. Data matching is defined as identifying the features that express the same entity in the real world from different data sets. Data updating refers to identifying the changed parts in the map and changing them to make the map closed to the reality. Data integrating refers to detecting the omitted features in the small-scale map and adding them to the map so that the new map has the same completeness as the large one. All in all, in the process of multi-scale thematic maps integration, coordinate system unifying is the basic step, data matching is the prerequisite, and data updating and integrating are the core steps and the destination. The following chat shows the framework of multi-scale thematic maps integration.

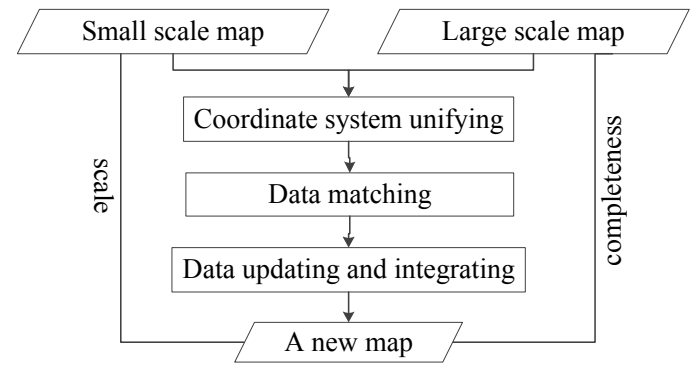

Figure 1: The framework of multi-scale thematic maps integration

The new map produced by the strategy proposed in this paper will have better usability. For example, when we need to analyze the spatial characteristics of the rivers in a region, using the small-scale river thematic map to analyze will acquire inaccurate results. Meanwhile, using the large-scale river thematic map to analyze will cost too much time because of its big memory size. At that time, if we use the new map to analyze, we can get more precise results and the time of data processing will be reduced to a certain extent. Therefore, this mapping method proposed in this paper is of great significance in the production and research fields.

\subsection{The Characteristics of Multi-scale River Thematic} Maps

As we all know, a river can be abstracted to a polyline or a polygon in a vector map, which means that the same river can be expressed as different feature forms in maps with various scales. For example, the Yangtze can be abstracted to a polyline in a small-scale map, such as 1:50,000 or 1:10,000, while it can be abstracted to a polygon in a large-scale map, such as 1:1,000 or 1:500. Different thematic maps of different scales can be used in different research fields.

Besides, on the large-scale river thematic maps, the characteristics and details of the river can be expressed as specific as possible; While in the small-scale river thematic maps, only the trunk and main tributaries of the river can be abstracted. Thus, compared with the small-scale map, the large-scale map is more complete in terms of features' quantity and more specific in terms of details, while the small-scale map takes up less storage than large-scale map. And this paper aims to put forward a strategy that can be used to produce a new map, and the new map would inherit the advantages of both the two kinds of maps. This paper only researched on the integration of multi-scale line 
river thematic maps.

\subsection{An Algorithm of Multi-scale Line Features Matching}

In this paper, we proposed an algorithm for multi-scale line features matching by calculating the distance from node to polyline. We regard the smaller scale map as reference layer and regard the larger scale map as target one. The principle of the algorithm is to traverse every feature on the reference layer, and find one or more matching polylines on the target layer. This algorithm can deal with the complex situation when a river is expressed as one polyline in the small-scale map, while it is divided into two or more polylines in the large-scale map. The following chat shows the flow chart of multi-scale line features matching:

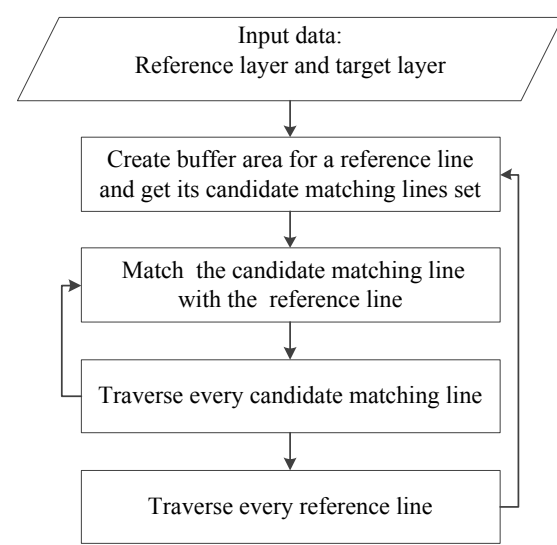

Figure 2: The flow chart of multi-scale line features matching

The specific steps are as follows:

(1) Create buffer area with radius $\mathbf{d}$ for feature $\mathbf{L}$ on the reference layer. For every reference feature $\mathbf{L}$, choose the target features that intersect with or be completely within reference feature L's buffer area and collect them together as candidate matching features set $\left(\mathrm{L}_{1}, \mathrm{~L}_{2} \ldots, \mathrm{L}_{\mathrm{m}}\right)$ of reference feature $\mathbf{L}$;

(2) For the feature $\mathbf{L}_{\mathbf{i}}$ in the candidate matching feature set, calculate the minimum distance $\mathbf{D}_{\mathbf{i}}(\mathrm{i}=1,2, \ldots \mathrm{n})$ from every point $\mathbf{P}_{\mathbf{i}}(\mathrm{i}=1,2, \ldots \mathrm{n})$ of the feature $\mathbf{L}_{\mathbf{i}}$ to the reference feature $\mathbf{L}$ and count the points whose minimum distances are shorter than the preset matching tolerance D_lim as $\mathbf{k}$. Finally, we get the ratio $\mathbf{f}$ of $\mathbf{k}$ to the total point number of the feature $\mathbf{L}_{\mathbf{i}}$;

(3) Set a threshold rate_lim. If $\mathrm{f}>$ rate_lim or $\mathbf{f}=$ rate_lim, it indicates that this candidate matching feature $\mathbf{L}_{\mathbf{i}}$ matches with the reference matching line $\mathbf{L}$;
(4) Traverse all candidate matching lines, and repeat the step(2) to step(3). Finally, we get all the target matching features of reference feature $\mathbf{L}$;

(5) Traverse all the reference features on the reference layer, and repeat the step(1) to step(4).

\subsection{A Strategy for Updating and integrating with Multi-} scale Thematic Maps

This paper put forward an integrating algorithm by simplifying, shortening and merging some features on original multi-scale thematic maps according to the matching results. The principle of the algorithm is to choose and deal with the proper features we want from the original multi-scale thematic maps and integrate them on a new map so that we can guarantee that the scale and completeness of the new map would meet our needs. The following chat shows the flow chart of multi-scale maps integrating:

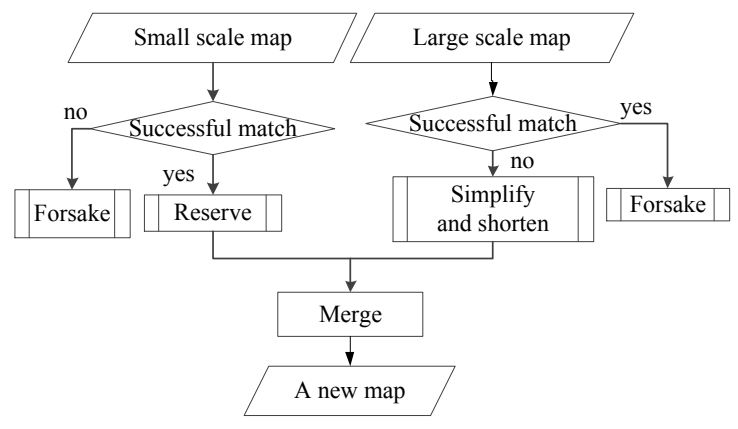

Figure 3: The flow chart of multi-scale maps updating

The specific steps are as follows:

(1) According to the matching results, find the features that exist both on the original small-scale and large-scale map, and extract them from the original small-scale map;

(2) Simplify and shorten the features that do not exist on the original small-scale map but exist on the large-scale map. In this paper, we use Douglas-Peucker algorithm to simplify the polylines;

(3) For features in step(2), after simplifying, we keep their shape unchanged and shorten them so that we can ensure that their scale would be consistent with the small-scale map. The principle of the feature shortening is as below: Supposing that the scale of large-scale map is $S_{1}$, the scale of small-scale map is $S_{2}$, the length of the feature to be shortened is L, the length of the feature after shortening is L', and we assure that the following equation could be established: 
Figure 5: The result of matching

$$
\frac{L^{\prime}}{L}=\frac{S_{2}}{S_{1}}
$$

(4) Merge the features in (1) and (3) into a same layer and a new map would be obtained. The characteristic of the new map is that its scale is as same as the small-scale map, its features are more complete, and its memory size is much smaller.

\section{EXPERIMENTAL RESULTS}

Three river thematic maps of Fengdianxiang, in Xinyang City of Henan Province were used for the integration experiments. The scales of these three maps are:1:10,000、1:50,000 and 1:100,000.The three maps are shown in Figure 4. According to Figure 4, we can find that the larger the map's scale is, and the more detailed the map is.

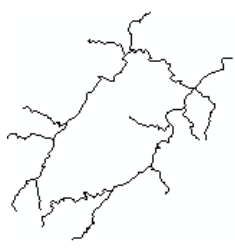

(a) $1: 100,000$

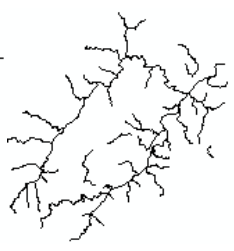

(b) $1: 50,000$

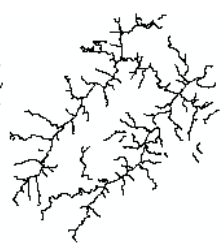

(c) $1: 10,000$

Figure 4: The original maps of different scales

In this paper, there are three decisive parameters for the multiscale feature matching algorithm and they are the buffer radius $\mathrm{d}$, the matching tolerance D lim and rate lim. Experimental results show that all the correct candidate lines could be chosen when the buffer radius $d$ is $15 \mathrm{~m}$ and almost all matching line on the target layer could be selected when the matching tolerance D_lim is $50 \mathrm{~m}$ and rate_lim is $80 \%$. The matching result for the 1:10,000 and 1:50,000 maps is shown in Figure 5. The left figure is the original 1:50,000 map and also the reference layer, while the right map is the matching result of 1:10,000 map. The red lines are the matched features on the target layer, while the green lines are the unmatched features on the target layer.

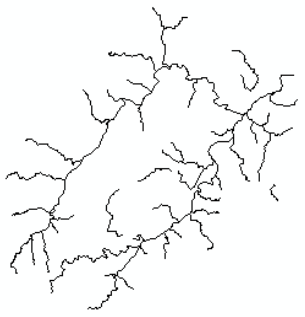

(a)

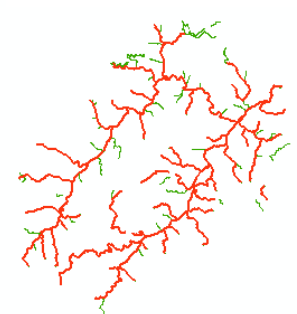

(b)
When 1:10,000 and 1:50,000 maps are integrated, we should simplify the chosen target features, keep their shape unchanged and shorten their lengths to $1 / 5$ of their original lengths to convert their scale from 1:10,000 to 1:50,000. Integrate any two maps of the three, and we got three groups of results. Table 1 shows the final results of this experiment. And the Table. 1 is at the last of the main body of this paper.

According to Table.1, compared with the original maps, the scale of the integrated map is as same as the original small-scale map, the features on the integrated map are as complete as the original large-scale map, and the memory size is much smaller than the original large-scale map. The experimental results showed that the strategy proposed in this paper is feasible and effective.

\section{CONCLUSIONS AND FUTURE WORK}

As we know, although using large-scale maps for spatial analysis could be more accurate, low computation efficiency will be a big problem due to its large data amount. In the other hand, using small-scale maps for spatial analysis would get inaccurate results because of its incomplete features. This paper proposed a new method for small-scale map generation, which can inherit as many necessary features as a small-scale map could contain, and reduce data amount simultaneously. The new generated map can not only speed up the analysis calculation, but also ensure the accuracy of the results.

Firstly, this paper summarized the basic framework of multiscale maps integration. There are three steps, namely coordinate system unifying, feature matching, data updating and integrating.

Secondly, this paper analyzed the differences of river thematic maps with different scales. The same river may be expressed diversely on the maps with different scales. And the larger the map's scale is, the more detailed the map is.

Thirdly, this paper put forward an algorithm for multi-scale line features matching by calculating the distance from node to polyline and an integrating algorithm by simplifying, shortening and merging the features on original multi-scale thematic maps.

Fourthly, to verify the feasibility and effectiveness of the method 
proposed in this paper, three river thematic maps with different scales were used for the integration experiments. According to the experimental results, we found that the scale of the integrated map was as same as the original small-scale map, and the feature quantity was almost as same as the original large-scale map, meanwhile its memory size is much smaller.
The deficiency of this paper is that it only focused on the method of integration for multi-scale line thematic maps. Following this direction, we can continue to research on the method of integration for multi-scale thematic maps of other types, such as the integration for point maps and integration for polygon maps. Ultimately, we can form a series of theory and system that can serve the production and research better.

Table 1: The results of three groups of integration

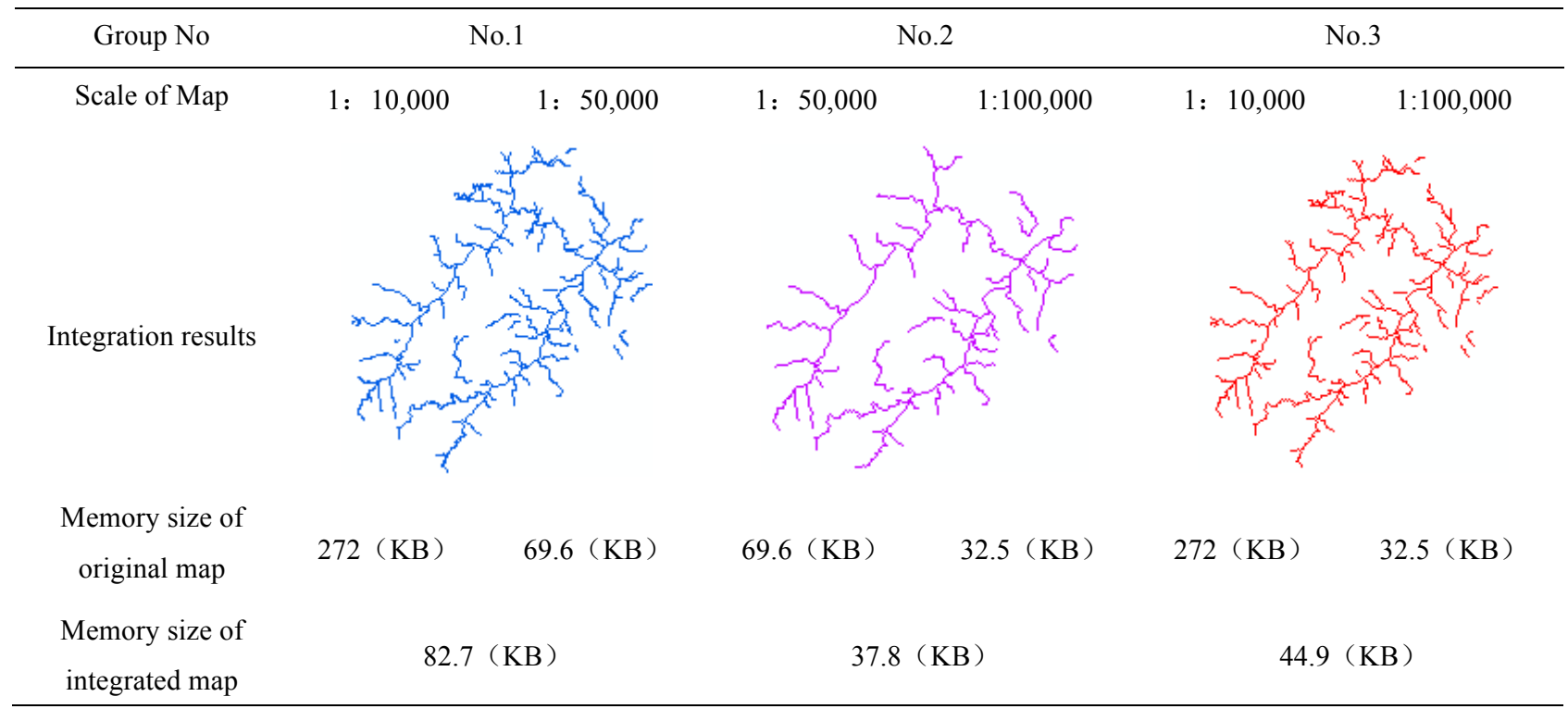

\section{REFERENCES}

Chen H., Liu D., Xu M., et al, 2013. Research on the Flowline and Status of Spatial Data Fusion. Geomatics World, 20(5), pp.26-31.

Chen Y., Gong J., Shi W., 2007. A Distance-based Matching Algorithm for Multi-scale Road Networks. Acta Geodaetica Et Cartographica Sinica, 36(1), pp.84-90.

Filin S. and Doysher Y, 1999. A liner Mapping Approach to Map conflation: Matching of Polylines. Surveying and Land Information Systems, 59(2), pp.107-114.

Fu Z., Wu J., 2007. Update Technologies for Multi-scale Spatial Database. Geomatics and Information Science of Wuhan University, 32(12), pp.1115-1119.

He J, Fei L, 2006. Three-dimensional Douglas-Peucker algorithm and the study on its application to automated generalization of DEM. Geoinformatics 2006: Geospatial Information Science. International Society for Optics and Photonics, pp.703-718.

Hu T., Mao Z., 2011. Methodological research on optional matching candidates of line objects. Science of Surveying and
Mapping 36(2), pp.132-135.

Lan Q., Li J, 2013. Research on Cascade Updating Methods for Multi-scale Map Data. Bulletin of Surveying and Mapping, (4),pp.33-36.

Lv X., Wu F, 2006. Representation of Topological Similarity Relationships for Spatial Objects in Multi Scale Representation. Journal of Geomatics 31(2), pp.39-31.

Mustière S, 2006. Results of experiments on automated matching of networks at different scales. International Archives of Photogrammetry, Remote Sensing and Spatial Information Sciences 36 (Part 2/W40), pp.92-100.

ST Wu, ACGD Silva, 2004. The Douglas-peucker algorithm: sufficiency conditions for non-self-intersections. Journal of the Brazilian Computer Society, 9(3), pp.67-84.

Walter, Fritsh, 1999. Matching Spatial Data Sets: A Statistical Approach. International Journal of Geographical information Systems 13(5), pp.445-473.

Xiao J., An X., Sun Q, et al. 2011. Research on automatic relating updating for multi-scale vector map database. Engineering of Surveying and Mapping, 20(2), pp.1-5. 